Applied Mathematical Sciences, Vol. 7, 2013, no. 97, 4821 - 4828

HIKARI Ltd, www.m-hikari.com

http://dx.doi.org/10.12988/ams.2013.36322

\title{
On the Shortest Common Parameterized Supersequence Problem
}

\author{
Anna Gorbenko \\ Department of Intelligent Systems and Robotics \\ Ural Federal University \\ 620083 Ekaterinburg, Russia \\ gorbenko.ann@gmail.com \\ Vladimir Popov \\ Department of Intelligent Systems and Robotics \\ Ural Federal University \\ 620083 Ekaterinburg, Russia \\ Vladimir.Popov@usu.ru
}

Copyright (c) 2013 Anna Gorbenko and Vladimir Popov. This is an open access article distributed under the Creative Commons Attribution License, which permits unrestricted use, distribution, and reproduction in any medium, provided the original work is properly cited.

\begin{abstract}
In this paper, we consider an approach to solve the problem of the shortest common parameterized supersequence. This approach is based on an explicit reduction from the shortest common parameterized supersequence problem to the 3-satisfiability problem and the maximum 2-satisfiability problem.
\end{abstract}

Keywords: 3-satisfiability problem, maximum 2-satisfiability problem, shortest common supersequence, parameterized supersequence, NP-complete

Investigation of various problems on sequences of symbols forms a fundamental part of computer science (see e.g. [1] - [6]). One of the most important problems in analysis of sequences is the shortest common supersequence problem. There are a large number of different variants of this problem. In 
particular, we can mention the shortest common parameterized supersequence problem [7]. In this paper, we consider an approach to solve the shortest common parameterized supersequence problem (SCPS). Note that encoding various hard problems as instances of the satisfiability problem and solving them with efficient satisfiability algorithms has caused considerable interest (see e.g. [8] - [23]). An explicit reduction from SCPS to the satisfiability problem was proposed in [7]. In this paper, we consider an explicit reduction from SCPS to the 3-satisfiability problem and the maximum 2-satisfiability problem (MAX2SAT).

Note that

$$
\begin{gathered}
\alpha \Leftrightarrow \quad\left(\alpha \vee \beta_{1} \vee \beta_{2}\right) \wedge\left(\alpha \vee \neg \beta_{1} \vee \beta_{2}\right) \wedge \\
\left(\alpha \vee \beta_{1} \vee \neg \beta_{2}\right) \wedge\left(\alpha \vee \neg \beta_{1} \vee \neg \beta_{2}\right), \\
\vee_{j=1}^{l} \alpha_{j} \Leftrightarrow \quad\left(\alpha_{1} \vee \alpha_{2} \vee \beta_{1}\right) \wedge \\
\left(\wedge_{i=1}^{l-4}\left(\neg \beta_{i} \vee \alpha_{i+2} \vee \beta_{i+1}\right)\right) \wedge\left(\neg \beta_{l-3} \vee \alpha_{l-1} \vee \alpha_{l}\right), \\
\alpha_{1} \vee \alpha_{2} \Leftrightarrow \quad\left(\alpha_{1} \vee \alpha_{2} \vee \beta\right) \wedge\left(\alpha_{1} \vee \alpha_{2} \vee \neg \beta\right), \\
\vee_{j=1}^{4} \alpha_{j} \Leftrightarrow \quad\left(\alpha_{1} \vee \alpha_{2} \vee \beta_{1}\right) \wedge\left(\neg \beta_{1} \vee \alpha_{3} \vee \alpha_{4}\right)
\end{gathered}
$$

where $l>4$.

Using relations $(1)-(4)$ we can obtain an explicit transformation of function $\xi$ from [7] in $\tau$ such that $\xi \Leftrightarrow \tau$ and $\tau$ is a 3-CNF. Clearly, $\tau$ give us an explicit reduction from SCPS to 3SAT.

Now we consider an explicit reduction from SCPS to MAX2SAT. In the decision version MAX2SAT can be formulated as following.

MAX2SAT:

INSTANCE: Given a set $U$ of variables, a collection $\mathbf{C}$ of clauses over $U$ such that each clause $\mathcal{C} \in \mathbf{C}$ has at most two literals, and a positive integer $k$.

QUESTION: Is there a truth assignment for $U$ that simultaneously satisfies at least $k$ of the clauses in $\mathbf{C}$ ?

Now, consider the following boolean function:

$$
\begin{gathered}
x \wedge y \wedge z \wedge w \wedge(\neg x \vee \neg y) \wedge(\neg y \vee \neg z) \wedge \\
(\neg z \vee \neg x) \wedge(x \vee \neg w) \wedge(y \vee \neg w) \wedge(z \vee \neg w)
\end{gathered}
$$


It is easy to check that there is no way to satisfy all these clauses. Note that these ten clauses have the following interesting property:

Any truth assignment that satisfies $x \vee y \vee z$ can be extended to satisfy seven of them and no more, while the remaining truth assignment can be extended to satisfy only six of them

(see e.g. [24], proof of theorem 9.2.).

Using this property we can construct $\eta$ by simply replacing in $\tau$ every occurrence of $x \vee y \vee z$ where $x, y$, and $z$ are some literals by (5) where $w$ is a new variable. It is easy to see that $\tau \Leftrightarrow \eta$. It is clear that $\eta$ give us explicit reduction from SCPS to MAX2SAT.

There is a well known site on which posted solvers for SAT [25]. In addition to the solvers the site also represented a large set of test problems. This set includes a randomly generated problems of 3SAT. We create a generator of natural instances for SCPS. Also we use test problems from [25].

We use algorithms fgrasp and posit from [25]. Also we use a simple genetic algorithm (SGA) and design our own genetic algorithm (OA) for SAT which based on algorithms from [25].

Consider a boolean function $g\left(x_{1}, x_{2}, \ldots, x_{n}\right)=\wedge_{i=1}^{m} \mathcal{C}_{i}$, where $m \geq 1$, and each of the $\mathcal{C}_{i}$ is the disjunction of one or more literals. Let $\left|\mathcal{C}_{i}\right|$ be a number of literals in $\mathcal{C}_{i}$. Let $\operatorname{occ}\left(x_{i}, g\right)$ be a number of occurrences of $x_{i}$ in $g$. Respectively, let $\operatorname{occ}\left(\neg x_{i}, g\right)$ be a number of occurrences of $x_{i}$ in $g$. For example, if $g=$ $\left(x_{1} \vee x_{2}\right) \wedge\left(\neg x_{2} \vee x_{3}\right) \wedge\left(x_{1} \vee x_{4}\right) \wedge\left(\neg x_{1} \vee x_{5}\right) \wedge\left(x_{1} \vee \neg x_{4}\right)$, then

$$
\begin{aligned}
& \operatorname{occ}\left(x_{1}, g\right)=3, \operatorname{occ}\left(\neg x_{1}, g\right)=1, \\
& \operatorname{occ}\left(x_{2}, g\right)=1, \operatorname{occ}\left(\neg x_{2}, g\right)=1, \\
& \operatorname{occ}\left(x_{3}, g\right)=1, \operatorname{occ}\left(\neg x_{3}, g\right)=0, \\
& \operatorname{occ}\left(x_{4}, g\right)=1, \operatorname{occ}\left(\neg x_{4}, g\right)=1, \\
& \operatorname{occ}\left(x_{5}, g\right)=1, \operatorname{occ}\left(\neg x_{5}, g\right)=0 .
\end{aligned}
$$

We consider a number of natural principles that define importance of a variable $x_{i}$ for satisfiability of boolean function $g$. These principles suggest us correct values of variables.

1. If

$$
\min _{\operatorname{occ}\left(x_{i}, \mathcal{C}_{j}\right)>0}\left|\mathcal{C}_{j}\right| \leq \min _{\operatorname{occ}\left(\neg x_{i}, \mathcal{C}_{j}\right)>0}\left|\mathcal{C}_{j}\right|
$$

then $x_{i}=1$. 
2. If

$$
\min _{\operatorname{occ}\left(x_{i}, \mathcal{C}_{j}\right)>0}\left|\mathcal{C}_{j}\right| \geq \min _{\operatorname{occ}\left(\neg x_{i}, \mathcal{C}_{j}\right)>0}\left|\mathcal{C}_{j}\right|
$$

then $x_{i}=0$.

3. Given positive integers $p_{1}, p_{2}, \ldots, p_{m}$. If

$$
\sum_{1 \leq j \leq m,\left|\mathcal{C}_{j}\right| \leq p_{j}} \operatorname{occ}\left(x_{i}, \mathcal{C}_{j}\right) \geq \sum_{1 \leq j \leq m,\left|\mathcal{C}_{j}\right| \leq p_{j}} \operatorname{occ}\left(\neg x_{i}, \mathcal{C}_{j}\right),
$$

then $x_{i}=1$.

4. Given positive integers $p_{1}, p_{2}, \ldots, p_{m}, q_{1}, q_{2}, \ldots, q_{m}$. If

$$
\sum_{1 \leq j \leq m,\left|\mathcal{C}_{j}\right| \leq p_{j}} \operatorname{occ}\left(x_{i}, \mathcal{C}_{j}\right) \geq \sum_{1 \leq j \leq m,\left|\mathcal{C}_{j}\right| \leq q_{j}} \operatorname{occ}\left(\neg x_{i}, \mathcal{C}_{j}\right),
$$

then $x_{i}=1$.

5. Given positive integers $p_{1}, p_{2}, \ldots, p_{m}, q_{1}, q_{2}, \ldots, q_{m}$ and a set of rational numbers

$$
\mathcal{Q}=\left\{\alpha_{i, u}, \beta_{i, v} \mid 1 \leq i \leq m, 1 \leq u \leq p_{i}, 1 \leq v \leq q_{i}\right\}
$$

If

$$
\sum_{1 \leq j \leq m, 1 \leq u \leq p_{j},\left|\mathcal{C}_{j}\right|=u} \alpha_{j, u} \operatorname{occ}\left(x_{i}, \mathcal{C}_{j}\right) \geq \sum_{1 \leq j \leq m, 1 \leq v \leq q_{j},\left|\mathcal{C}_{j}\right|=v} \beta_{j, v} \operatorname{occ}\left(\neg x_{i}, \mathcal{C}_{j}\right),
$$

then $x_{i}=1$.

Based on these principles, we can consider the following five types of commands:

$$
\begin{gathered}
P_{1}, P_{2}, P_{3}\left[p_{1}, p_{2}, \ldots, p_{m}\right], P_{4}\left[p_{1}, p_{2}, \ldots, p_{m}, q_{1}, q_{2}, \ldots, q_{m}\right] \\
P_{5}\left[p_{1}, p_{2}, \ldots, p_{m}, q_{1}, q_{2}, \ldots, q_{m}, \mathcal{Q}\right] .
\end{gathered}
$$

Also we consider the following three commands for run algorithms: Try_fgrasp, Try_posit, and Try_ga, where Try_ga runs a simple genetic algorithm. Denote by $\mathcal{R}$ the set of commands of these eight types. Arbitrary element of $\mathcal{R}^{*}$ it is possible to consider as a program for finding values of variables of a boolean function. We assume that such programs are executed on a cluster. Execution of each of commands of type $P_{i}$ reduces the number of variables of a boolean function by one. Execution of each of commands Try_fgrasp, Try_posit, and Try_ga consists in the run of corresponding algorithm for current 
boolean function on a separate set of calculation nodes and the transition to the next command. Algorithms fgrasp [25] and posit [25] we run only on one calculation node. Genetic algorithms can be used in parallel execution. We use auxiliary genetic algorithms which determine the number of calculation nodes and, if necessary, scale commands $P_{3}, P_{4}, P_{5}$.

Initially, we selected a random subset of $\mathcal{R}^{*}$. We use a genetic algorithm to select a program from the current subset of $\mathcal{R}^{*}$ and a genetic algorithm for evolving the current subset of $\mathcal{R}^{*}$. The evolution of the current subset of $\mathcal{R}^{*}$ implemented on a separate set of calculation nodes. For every subsequent boolean functions it is used the current subset of $\mathcal{R}^{*}$ which is obtained by taking into account the results of previous runs.

We use heterogeneous cluster based on three clusters (Cluster USU, Linux, 8 calculation nodes; umt, Linux, 256 calculation nodes; um64, Linux, 124 calculation nodes) [26]. Each test was run on a cluster of at least 100 nodes.

Algorithms fgrasp and posit used only for 3SAT. Respectively, for SAT and MAX2SAT we use only simple genetic algorithm (SGA), and our algorithm (OA). Selected experimental results for 3SAT, SAT, and MAX2SAT are given in Tables 1 and 2 .

\begin{tabular}{|l|llll|}
\hline time & fgrasp & posit & SGA & OA \\
\hline average & $31.8 \mathrm{~min}$ & $33.9 \mathrm{~min}$ & $37.4 \mathrm{~min}$ & $2.7 \mathrm{~min}$ \\
$\max$ & $22.8 \mathrm{~h}$ & $26.1 \mathrm{~h}$ & $28 \mathrm{~h}$ & $5 \mathrm{~h}$ \\
best & $1.4 \mathrm{~min}$ & $45 \mathrm{sec}$ & $19 \mathrm{sec}$ & $17 \mathrm{sec}$ \\
\hline
\end{tabular}

Table 1: Experimental results for 3SAT

\begin{tabular}{|l|llll|}
\hline time & SGA & OA & SGA & OA \\
& SAT & SAT & MAX2SAT & MAX2SAT \\
\hline average & $32.5 \mathrm{~min}$ & $28.9 \mathrm{~min}$ & $39.3 \mathrm{~min}$ & $4.8 \mathrm{~min}$ \\
$\max$ & $27.1 \mathrm{~h}$ & $21.2 \mathrm{~h}$ & $43.4 \mathrm{~h}$ & $9.1 \mathrm{~h}$ \\
best & $23 \mathrm{sec}$ & $19 \mathrm{sec}$ & $11 \mathrm{sec}$ & $21 \mathrm{sec}$ \\
\hline
\end{tabular}

Table 2: Experimental results for SAT and MAX2SAT

It is interesting to note that the performance of OA depends essentially on the number of letters from $\Pi$ (see e.g. Table 3 and 4 ).

In experiments presented in Tables $1-4$ OA used after 10000 runs. The dependence of the performance of OA on the number of runs is shown in Table 5 .

In this paper we describe an approach to solve the problem SCPS. This approach is based on constructing logical models for SCPS. Also we consider 


\begin{tabular}{|l|llll|}
\hline time & $10 \%$ & $30 \%$ & $60 \%$ & $90 \%$ \\
\hline average & $17.2 \mathrm{~min}$ & $2.1 \mathrm{~min}$ & $1.9 \mathrm{~min}$ & $8.4 \mathrm{~min}$ \\
max & $4.6 \mathrm{~h}$ & $2.6 \mathrm{~h}$ & $56 \mathrm{~min}$ & $5 \mathrm{~h}$ \\
best & $41 \mathrm{sec}$ & $17 \mathrm{sec}$ & $20 \mathrm{sec}$ & $18 \mathrm{sec}$ \\
\hline
\end{tabular}

Table 3: Experimental results for 3SAT runs of OA depending on the number of letters from $\Pi$

some genetic and local search algorithms for the problem. Our experimental results showed that our reduction to 3SAT with SAT-solver can be used as a test bed for intelligent algorithms for SCPS.

The main question that is of interest for future research is to study the dependence of complexity of solution on the distribution of letters from $\Pi$.

ACKNOWLEDGEMENTS. The work was partially supported by Analytical Departmental Program "Developing the scientific potential of high school", RFBR, research project No. 13-01-00048 a, and Ural Federal University development program with the financial support of young scientists.

\begin{tabular}{|l|llll|}
\hline time & $10 \%$ & $30 \%$ & $60 \%$ & $90 \%$ \\
\hline average & $41.4 \mathrm{~min}$ & $23.2 \mathrm{~min}$ & $24.5 \mathrm{~min}$ & $36.3 \mathrm{~min}$ \\
$\max$ & $19.6 \mathrm{~h}$ & $14.4 \mathrm{~h}$ & $17.8 \mathrm{~h}$ & $21.2 \mathrm{~h}$ \\
best & $25 \mathrm{sec}$ & $19 \mathrm{sec}$ & $20 \mathrm{sec}$ & $23 \mathrm{sec}$ \\
\hline
\end{tabular}

Table 4: Experimental results for SAT runs of OA depending on the number of letters from $\Pi$ 


\begin{tabular}{|l|llll|}
\hline time & 100 & 500 & 1000 & 5000 \\
\hline average & $44.6 \mathrm{~min}$ & $35.8 \mathrm{~min}$ & $33.6 \mathrm{~min}$ & $5.3 \mathrm{~min}$ \\
$\max$ & $29.2 \mathrm{~h}$ & $27.1 \mathrm{~h}$ & $15.4 \mathrm{~h}$ & $6.4 \mathrm{~h}$ \\
best & $11.8 \mathrm{~h}$ & $4.3 \mathrm{~min}$ & $26 \mathrm{sec}$ & $17 \mathrm{sec}$ \\
\hline
\end{tabular}

Table 5: Experimental results for 3SAT runs of OA depending on the number of runs

\section{References}

[1] V. Yu. Popov, Computational complexity of problems related to DNA sequencing by hybridization, Doklady Mathematics, 72 (2005), 642-644.

[2] V. Popov, The approximate period problem for DNA alphabet, Theoretical Computer Science, 304 (2003), 443-447.

[3] V. Popov, The Approximate Period Problem, IAENG International Journal of Computer Science, 36 (2009), 268-274.

[4] V. Popov, Approximate Periods of Strings for Absolute Distances, Applied Mathematical Sciences, 6 (2012), 6713-6717.

[5] V. Popov, Sorting by prefix reversals, IAENG International Journal of Applied Mathematics, 40 (2010), 247-250.

[6] V. Popov, Multiple genome rearrangement by swaps and by element duplications, Theoretical Computer Science, 385 (2007), 115-126.

[7] A. Gorbenko and V. Popov, The Shortest Common Parameterized Supersequence Problem, Applied Mathematical Sciences, 7 (2013), 2373-2380.

[8] A. Gorbenko and V. Popov, The Problem of Finding Two Edge-Disjoint Hamiltonian Cycles, Applied Mathematical Sciences, 6 (2012), 6563-6566.

[9] A. Gorbenko and V. Popov, Footstep Planning for Humanoid Robots, Applied Mathematical Sciences, 6 (2012), 6567-6571.

[10] A. Gorbenko and V. Popov, Hamiltonian Alternating Cycles with Fixed Number of Color Appearances, Applied Mathematical Sciences, 6 (2012), 6729-6731.

[11] A. Gorbenko and V. Popov, Task-resource Scheduling Problem, International Journal of Automation and Computing, 9 (2012), 429-441. 
[12] A. Gorbenko and V. Popov, Longest Common Parameterized Subsequences with Parameterized Common Substring, Applied Mathematical Sciences, 7 (2013), 2341-2345.

[13] A. Gorbenko and V. Popov, The Minimum k-Cover Problem, Applied Mathematical Sciences, 7 (2013), 2347-2352.

[14] A. Gorbenko and V. Popov, The Shortest Common Superstring Problem, Applied Mathematical Sciences, 7 (2013), 2353-2356.

[15] A. Gorbenko and V. Popov, Computational Experiments for the Problem of Footstep Planning for Humanoid Robots, Applied Mathematical Sciences, 7 (2013), 2357-2372.

[16] A. Gorbenko and V. Popov, The Minimum Test Collection Problem, Applied Mathematical Sciences, 7 (2013), 1191-1193.

[17] A. Gorbenko and V. Popov, The Farthest Substring Problem, Applied Mathematical Sciences, 7 (2013), 1209-1212.

[18] A. Gorbenko and V. Popov, The Swap Common Superstring Problem, Applied Mathematical Sciences, 7 (2013), 609-614.

[19] A. Gorbenko and V. Popov, The String Barcoding Problem, Applied Mathematical Sciences, 7 (2013), 615-622.

[20] A. Gorbenko and V. Popov, The c-Fragment Longest Arc-Preserving Common Subsequence Problem, IAENG International Journal of Computer Science, 39 (2012), 231-238.

[21] A. Gorbenko and V. Popov, The set of parameterized k-covers problem, Theoretical Computer Science, 423 (2012), 19-24.

[22] A. Gorbenko and V. Popov, On the Problem of Placement of Visual Landmarks, Applied Mathematical Sciences, 6 (2012), 689-696.

[23] A. Gorbenko and V. Popov, The Longest Common Parameterized Subsequence Problem, Applied Mathematical Sciences, 6 (2012), 2851-2855.

[24] C. H. Papadimitriou, Computational complexity, Addison Wesley, Reading, CA, 1994.

[25] http://people.cs.ubc.ca/ hoos/SATLIB/index-ubc.html

[26] http://parallel.imm.uran.ru/mvc_now/hardware/ supercomp.htm

Received: June 15, 2013 